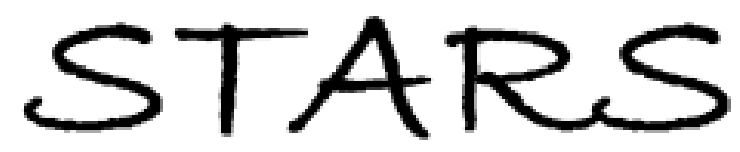

University of Central Florida

STARS

$1-1-2004$

\title{
Time- and spectrally resolved ultrafast gain dynamics of a semiconductor optical amplifier under phase-correlated multiwavelength pulse amplification
}

Luis C. Archundia

University of Central Florida

Bojan Resan

Peter J. Delfyett Jr.

Find similar works at: https://stars.library.ucf.edu/facultybib2000

University of Central Florida Libraries http://library.ucf.edu

This Article is brought to you for free and open access by the Faculty Bibliography at STARS. It has been accepted for inclusion in Faculty Bibliography 2000s by an authorized administrator of STARS. For more information, please contact STARS@ucf.edu.

\section{Recommended Citation}

Archundia, Luis C.; Resan, Bojan; and Delfyett, Peter J. Jr., "Time- and spectrally resolved ultrafast gain dynamics of a semiconductor optical amplifier under phase-correlated multiwavelength pulse amplification" (2004). Faculty Bibliography 2000s. 4190.

https://stars.library.ucf.edu/facultybib2000/4190

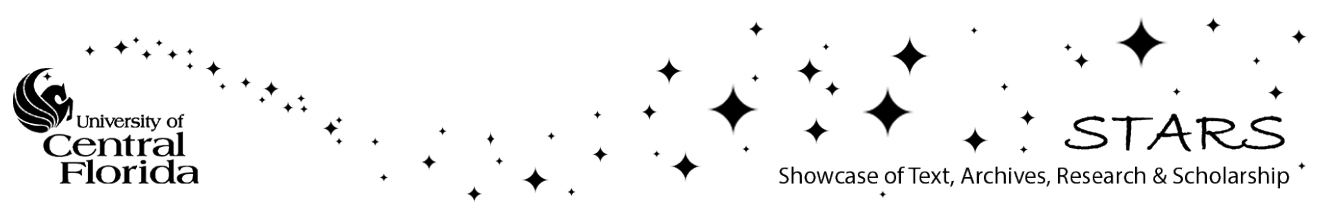




\title{
Time- and spectrally resolved ultrafast gain dynamics of a semiconductor optical amplifier under phase-correlated multiwavelength pulse amplification
}

\author{
Luis C. Archundia, ${ }^{\text {a) }}$ Bojan Resan, and Peter J. Delfyett, Jr. \\ CREOL \& FPCE/College of Optics and Photonics, University of Central Florida, \\ 4000 Central Florida Boulevard, Orlando, Florida 32816
}

(Received 14 June 2004; accepted 17 September 2004)

\begin{abstract}
The ultrafast gain dynamics of an $\mathrm{AlGaAs}$ semiconductor optical amplifier (SOA) are measured under phase-correlated multiwavelength pulse amplification using time-resolved pump-probe techniques. Both the temporal and spectral gain dynamics are measured. Carrier heating due to two photon absorption, carrier cooling, four-wave mixing, and cross-phase modulation effects are observed. These effects are evident when amplifying dispersion compensated pulses, and it is shown how these effects decrease when amplifying nondispersion compensated (chirped) pulses. This helps to avoid nonlinear effects in the gain media (SOA), which, in turn, helps to support the operation of external cavity multiwavelength semiconductor mode-locked lasers where the intracavity pulses are inherently chirped. (C) 2004 American Institute of Physics. [DOI: 10.1063/1.1823037]
\end{abstract}

Multiwavelength sources are important for optical networks and signal processing where wavelength division multiplexing is utilized to increase the bit rate. ${ }^{\text {,2 }}$ It has recently been shown that multiwavelength mode-locked semiconductor lasers have proven to be attractive candidates for such applications. ${ }^{3}$ As an effort to better understand the mechanisms that support the operation of multiwavelength modelocked semiconductor lasers, we use time-resolved pumpprobe techniques to measure the ultrafast gain dynamics of a semiconductor optical amplifier under multiwavelength pulse amplification. These results show that nonlinear effects such as carrier heating and cooling, four-wave mixing, and selfphase modulation, ${ }^{4}$ can be controlled by tailoring the chirp between each wavelength channel.

The device under test is an AlGaAs semiconductor optical amplifier (SOA) biased at a constant current of $200 \mathrm{~mA}^{5}$ The SOA is $350 \mu \mathrm{m}$ long and is angle striped and antireflection coated to avoid reflection from the facets. The experimental setup is shown in Fig. 1. An external cavity semiconductor mode-locked laser is used as the pulse source for the pump-probe measurements. The laser produces optical pulses of $6 \mathrm{ps}$ in duration at a repetition rate of $271 \mathrm{MHz}$, with a spectral bandwidth of approximately $4 \mathrm{~nm}$, centered at $835 \mathrm{~nm}$, with $4 \mathrm{~mW}$ of average output power. The pulses are primarily linearly chirped, ${ }^{6}$ and can be externally compressed using a dispersion compensator.

Most of the laser power is used for the pump and a small part is used for the probe. The probe pulse duration is reduced to $\sim 750 \mathrm{fs}$ using a dual grating dispersion compensator. ${ }^{7}$ The probe polarization is rotated $90^{\circ}$ and a variable filter is used to adjust its power. A variable delay station is used to delay the probe with respect to the pump.

The pump is obtained by passing the laser pulses through a second dual grating dispersion compensator where the linear chirp is compensated and the spectrum is filtered to generate three wavelength channels of approximately $0.5 \mathrm{~nm}$ full width at half maximum each, with a $1.4 \mathrm{~nm}$ channel to channel separation. Since the pump is comprised of three

\footnotetext{
${ }^{a)}$ Electronic mail: larchund@mail.ucf.edu
}

phase correlated wavelength channels, the temporal profile is a burst of short pulses under a broad envelope. The temporal duration of a single pulse within the pulse burst is related to the full spectral width encompassing all the wavelength channels, while the temporal duration of the pulse burst is related to the spectral bandwidth of a single wavelength channel. The separation between pulses within the pulse burst is related to the separation between wavelength channels.

The pump and the probe are coupled into the device under test, and the amplified probe power and spectrum are measured as a function of the delay between pump and probe. The strong pump pulses induce changes in the gain of the SOA, and the short probe pulses, which are sufficiently weak such that no significant changes in the gain are induced, measure the gain changes originated by the pump as a function of the delay between pump and probe. Crosspolarized pump and probe and lock-in detection techniques are used to help distinguish between pump and probe after being amplified by the SOA. ${ }^{6}$

Figures 2(a) and 2(b) show the pump spectrum before and after being amplified by the SOA. The salient feature of

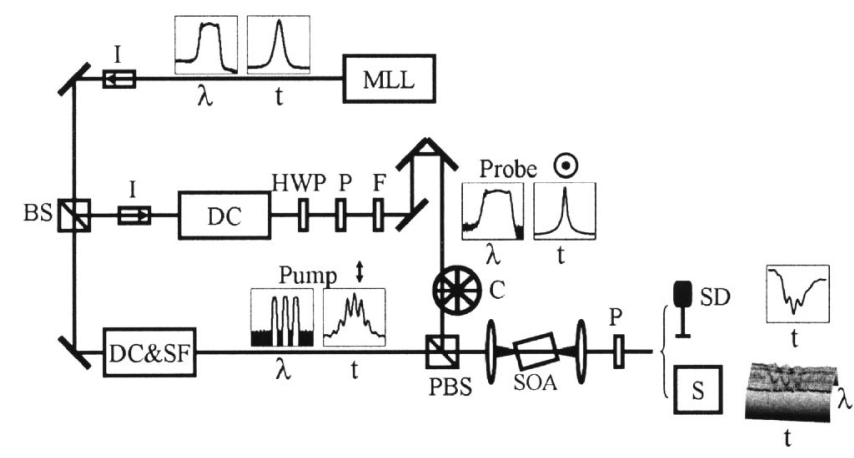

FIG. 1. Experimental setup. I-isolator; MLL-mode-locked laser; BSbeam splitter; DC—dispersion compensator; HWP—half wave plate; $\mathrm{P}$-polarizer; F-filter; $\mathrm{C}$-chopper; SF-spectral filter; PBS-polarization beam splitter; SOA—semiconductor optical amplifier under test; SD—slow detector; S-spectrometer. 

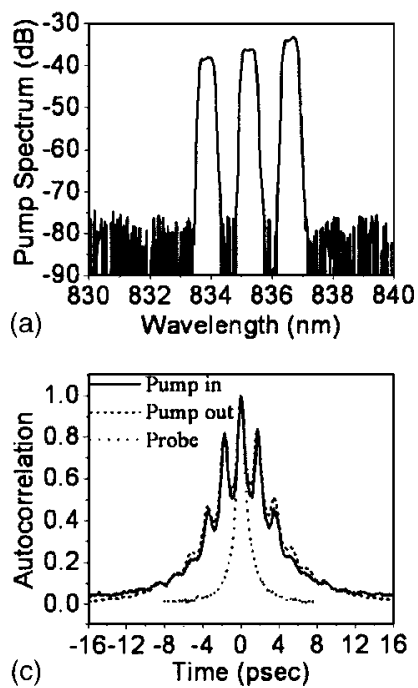

(b)
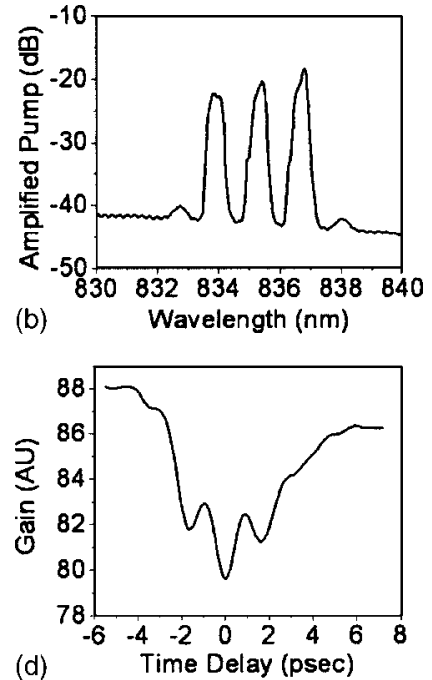

FIG. 2. Gain dynamics for dispersion compensated pulses. (a) Pump spectrum, (b) pump spectrum after amplification, (c) pump, probe, and pump after amplification autocorrelations, (d) time-resolved gain dynamics.

Fig. 2(b) is the additional wavelength channels generated by the multiwavelength pump pulse burst due to four-wave mixing in the $\mathrm{SOA}^{8}{ }^{8}$ suggesting the interchange of energy between wavelength channels, which also lead to a small spectral and temporal reshaping of the pulse burst. Figure 2(c) shows the pump and probe autocorrelations and Fig. 2(d) plots the probe signal as a function of the delay between the pump and the probe, which represents the SOA gain dynamics as the pump is amplified. In Fig. 2(d) a net reduction in the gain due to stimulated emission is observed for time delays greater than $6 \mathrm{ps}$, which recovers on a nanosecond time scale. In addition, for delays between -4 and $4 \mathrm{ps}$, a rapid variation of the gain is measured, which is caused by the periodic temporal nature of the phase coherent three wavelength pulse burst. These transients are related to two photon absorption (fast gain reduction) and the relaxation of hot carriers generated by two photon absorption (fast gain recovery). ${ }^{4}$

The time varying gain coupled to the refractive index results in an instantaneous frequency proportional to $-\partial n(t) / \partial t$, where $n(t)$ is the time varying refractive index. ${ }^{9}$ Figure 3(a) shows the gain dynamics of the SOA and Figs. 3(b) and 3(c) are qualitative representations of the index of refraction and the instantaneous frequency due to self-phase modulation.

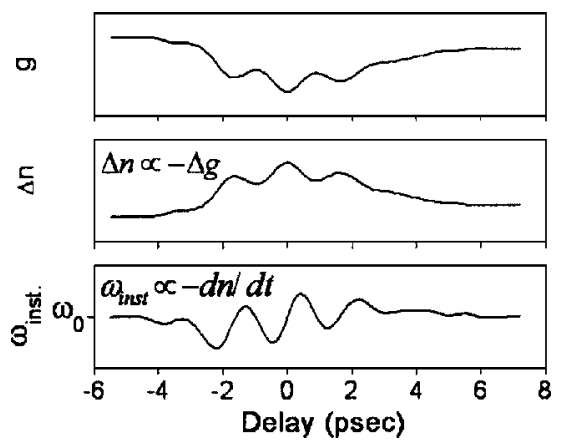

FIG. 3. Self-phase modulation. (a) SOA gain dynamics, (b) index of refraction dynamics, and (c) instantaneous frequency.

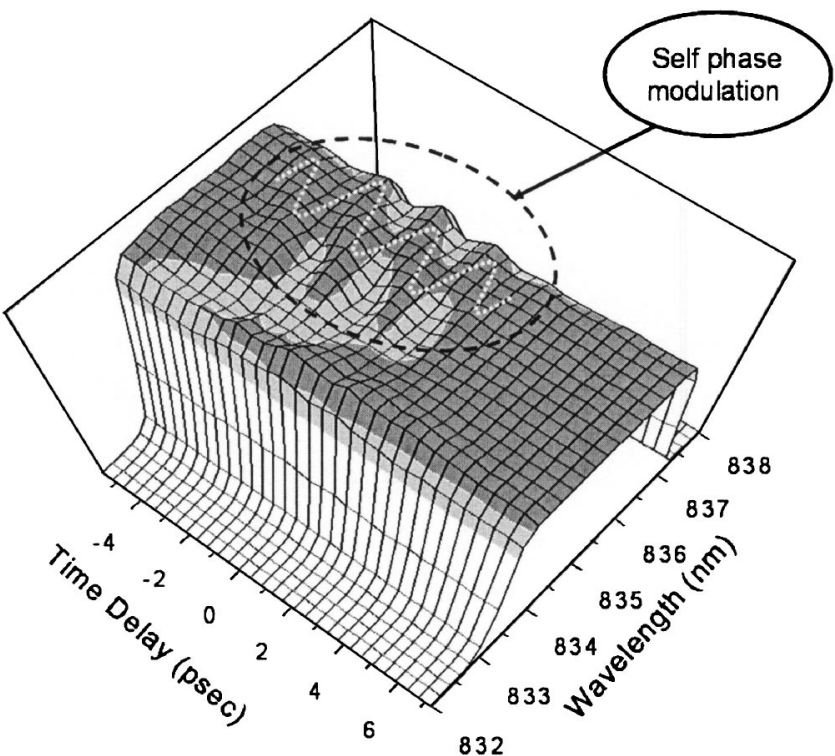

FIG. 4. Probe spectrum measured as a function of the delay between the pump and the probe for dispersion compensated pulses.

Owing to the large coupling between gain, carrier concentration, and refractive index, the rapid gain variations will induce rapid temporal variations in index and cause a phase modulation that will couple to the probe pulse via degenerate cross phase modulation. To observe this effect, a surface plot of the probe spectrum is measured as a function of the delay between the pump and the probe (see Fig. 4). In this figure, the probe spectra are normalized with respect to the gain of the SOA to observe only the spectral changes, not the gain changes. Also, the probe spectra are normalized with respect to the unperturbated probe spectrum, to yield a flattop spectrum as a reference. Changes in the spectrum of the probe due to cross-phase modulation induced by the pump are observed, where the peak wavelength periodically alternates between long and short wavelengths as shown by the white line in Fig. 4.

If Fig. 4 is analyzed with care and compared with Fig. 3, it can be observed that the long wavelength peaks correspond
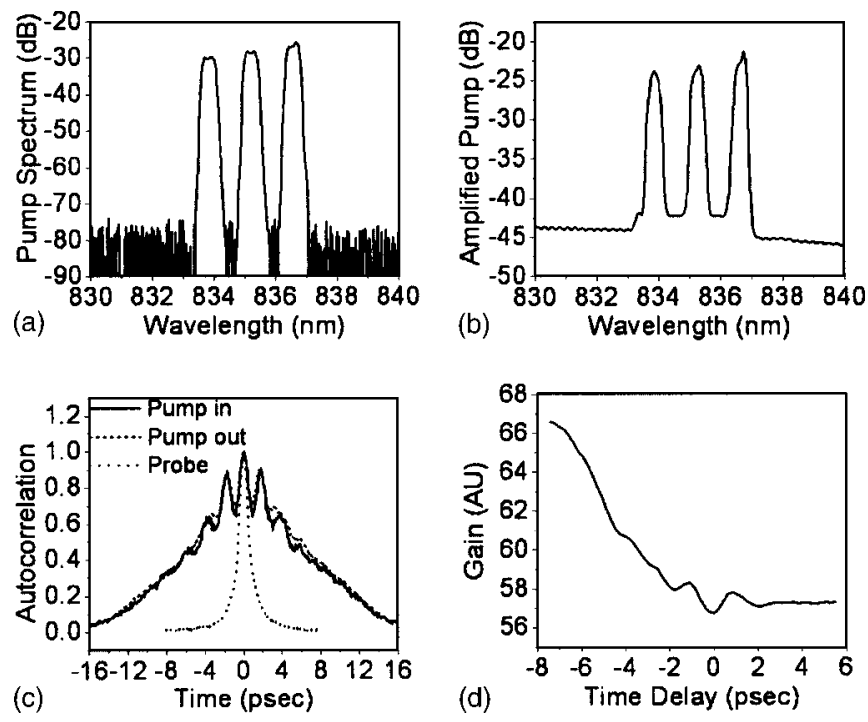

FIG. 5. Gain dynamics for nondispersion compensated pulses. (a) Pump spectrum, (b) pump spectrum after amplification, (c) pump, probe, and pump after amplification autocorrelations, (d) time-resolved gain dynamics. 


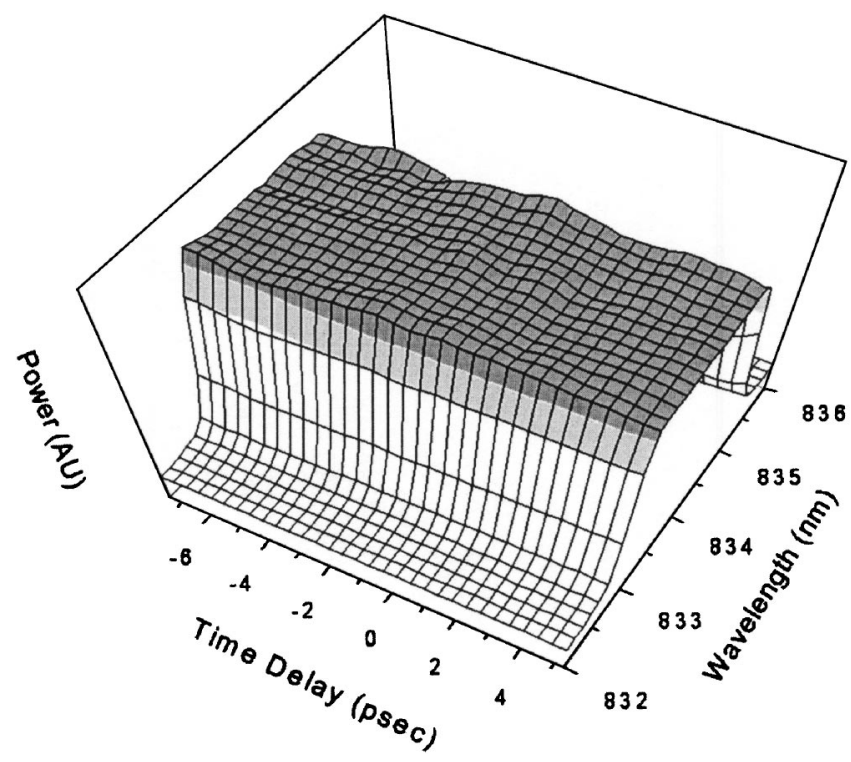

FIG. 6. Probe spectrum measured as a function of the delay between the pump and the probe for nondispersion compensated pulses.

to times when the gain is decreasing $(\Delta \omega<0)$ and the short wavelength peaks correspond to times when the gain is recovering $(\Delta \omega>0)$. This plot clearly shows the temporal evolution of the phase modulation induced on a weak probe pulse by a strong multiwavelength pump pulse.

The experiment was repeated with a nondispersion compensated pump. Experimentally, this is achieved by not compensating for the chirp impressed on the pulses generated from the mode-locked source. The spectral filtering employed to create the pump pulse in this case produces three mode-locked wavelength channels, but with a temporal skew, or delay, between each channel. This means that pulses do not coincide temporally, similar to observations in multiwavelength semiconductor mode-locked lasers, where the pulses from different wavelength channels are not temporally coincident. ${ }^{10}$ In addition, because the chirp is not compensated, the pump is broader in time, similar also to multiwavelength semiconductor mode-locked lasers, where broad chirped pulses are obtained. The results are shown in Figs. 5 and 6 . Figure 5 shows the input and output spectra, intensity autocorrelations, and the time-resolved gain, and Fig. 6 shows the time-resolved probe spectrum.

Comparing Figs. 5 and 6 with Figs. 2 and 4, it can be observed that the four-wave mixing effect is greatly suppressed. Note that the pump autocorrelation is broader with less temporal modulation owing to the temporal skew and the pulse broadening due to chirp. This leads to a slower gain depletion with less modulation, resulting in the reduction of the carrier heating and carrier cooling effects and better power extraction from the SOA as observed in Fig. 5(d). In addition, the induced cross phase modulation effects are no longer evident in Fig. 6.
Although long wavelengths lead short wavelengths in time, stronger amplification of the leading wavelengths is not observed in Fig. 5(b). Note that, while there is a temporal skew between wavelength channels, there is still a residual temporal overlap. Also, each wavelength channel is broader due to the uncompensated chirp resulting in a gain approximately equal for each wavelength channel. Additionally, the total gain change is approximately $15 \%$, and Fig. 5(b) is plotted in logarithmic scale making it hard to observe small changes.

In general, all the nonlinear effects were decreased when amplifying multiwavelength chirped pulses with small temporal skew imparted between each wavelength. This is owing to the fact that the temporal beating induced by the multiwavelength dispersion compensated pulses induce nonlinear effects that cause an additional periodic gain reduction, while the multiwavelength pulse with temporal skew and dispersion generates an optical pulse profile that does not generate temporal beats and therefore induces a temporal gain dynamic that extracts more energy from the SOA and reduces nonlinear effects. These time-resolved gain dynamics clearly illustrate the fundamental physical phenomena underlying the production of multiwavelength pulses from mode-locked semiconductor diode lasers.

In summary, time- and spectrally resolved ultrafast gain dynamics of a semiconductor optical amplifier were measured for the amplification of phase-correlated multiwavelength pulses. Four-wave mixing, carrier heating via two photon absorption, carrier cooling, and phase modulation effects were observed and it was shown that small temporal delays between mode-locked wavelength channels helps to avoid these nonlinear effects in the gain media (SOA), and support the operation of multiwavelength semiconductor mode-locked lasers.

This work was supported by National Science Foundation Grant No. ECS011338.

${ }^{1}$ L. Boivin, M. Wegmueller, and M. C. Nuss, IEEE Photonics Technol. Lett. 11, 466 (1999).

${ }^{2}$ O. Boyraz, J. Kim, M. N. Islam, F. Coppinger, and B. Jalali, J. Lightwave Technol. 18, 2167 (2000).

${ }^{3}$ M. Mielke, G. A. Alphonse, and P. J. Delfyett, IEEE Photonics Technol. Lett. 15, 501 (2003).

${ }^{4}$ K. L. Hall, G. Lenz, A. M. Darwish, and E. P. Ippen, Opt. Commun. 111, 589 (1994)

${ }^{5}$ G. A. Alphonse, D. B. Gilbert, M. G. Harvey, and M. Ettenberg, IEEE J. Quantum Electron. 24, 2454 (1988).

${ }^{6}$ P. J. Delfyett, L. T. Florez, N. Stoffel, T. Gmitter, N. C. Andeadakis, Y. Silberberg, J. P. Heritage, and G. A. Alphonse, IEEE J. Quantum Electron. 28, 2203 (1992)

${ }^{7}$ J.-C. Diels and W. Rudolph, Ultrafast Laser Pulse Phenomena. Fundamentals, Techniques, and Applications on a Femtosecond Time Scale (Academic, San Diego, 1996).

${ }^{8}$ H. Shi, I. Nitta, A. Schober, and P. J. Delfyett, Opt. Lett. 24, 238 (1999).

${ }^{9}$ G. P. Agrawal and N. A. Olsson, IEEE J. Quantum Electron. 25, 2297 (1989).

${ }^{10}$ M. Mielke and P. J. Delfyett, Opt. Lett. 27, 1064 (2002). 